\title{
A Study of Apparel Sizing System for Korean Obese Women
}

\author{
Kyong-Hwa Yi \\ Dept. of Clothing \& Textiles, The Catholic University of Korea \\ Received November 12, 2009; Revised December 10, 2009; Accepted December 15, 2009 \\ 비만여성의 의복치수체계 연구 \\ 이 경 화 \\ 가톨릭대학교 의류학 전공 \\ 접수일(2009년 11월 12일), 수정일(2009년 12월 10일), 게재확정일(2009년 12월 15일)
}

\begin{abstract}
This study suggests apparel sizing systems for obese women in their twenties to fifties. Size Korea body measurement data were used for sampling the obese subjects by obesity judgment indices. A total of 7 obesity judgment indices frequently used in the clothing field were inspected and the measurement data of 499 females defined as "obese" by over 5 obesity judgment indices were analyzed. The results of the study were as follows: A total of 2,212 female body measurement data for the Size Korea Project were collected. These were compared with the 499 data of the obese women in the study. Except for all heights and some lengths correlating with heights, the measurements of obese women were larger than the entire group of females. Bust girth, hip girth, and height were selected as control dimensions of the outerwear covering the upper body; waist girth and hip girth were selected as control dimensions of the outerwear covering the lower body. In each crosstabulation table, $3 \mathrm{~cm}$ pitches and $5 \mathrm{~cm}$ pitches by bust girth, waist girth, and hip girth were compared in the aspects of coverage and coverage efficiency. Finally, sizing charts for outerwear covering the upper body and the lower body of obese women were established in the study through the comparison of the coverage and the coverage efficiency. These Size charts were given by $5 \mathrm{~cm}$ pitches as final sizing systems. Size number of outerwear covering the upper body was 10 and size number of outerwear covering the lower body was 9 . In addition, the body measurements of control dimensions and referable dimensions for making garments are given.
\end{abstract}

Key words: Size, Sizing system, Obese women, Obesity; 치수, 치수체계, 비만여성, 비만

\section{Introduction}

According to the report submitted by the National Health Insurance Corp. (2009), 3.24 million, or $32.8 \%$, out of 9.87 million adults who had regular checkups in 2008 were obese. The report showed

Corresponding author

E-mail: ykh@catholic.ac.kr

This work was supported by the Catholic University of Korea, Research Fund, 2009. those categorized as obese had body mass index (BMI) measurements of 25 or higher. According to the report, the ratio of Korean adults with BMI readings of 25 or higher stood at $24.1 \%$ in 2007. The number of adults with BMI readings of 40 or higher, which indicates excess body fat, was 23,613 in 2008, compared with 7,020 in 2007 and 7,957 in 2006. Especially, young people in their twenties have shown a $75 \%$ increase in such complications during the same period, indicating a general health deteriora- 
tion compared to previous generations (NHIC, 2009). In the case of adult females, they are getting more obese according as they get older. In the results of the national health \& nutrition survey (NHIC, 2009), 31.7\% of the 4,600 people surveyed in 2007 were considered obese. $36.2 \%$ of men fell under the category of obesity, compared to $26.3 \%$ for women. Namely, the obesity problem in female adults is much more serious than in males. With the rise in obesity, the indices of obesity-related illnesses will rise accordingly. Also, as obesity rate grows, many obese people lose their chance to choose the right products for them. In particular, they have big difficulty to find suitable garments that fit. Recently, several apparel brands for obese women have launched as the number of obese people is increasing. There is not enough variety to choose from compared with apparel brands producing the normal-sized wears. In particular, body measurements are the most fundamental and valuable data that are necessary in clothing manufacture. Nonetheless, many of the obese subjects are not willing to have their measurements taken by the researchers. Therefore, there is no proper data obtained from obese subjects in the fashion industry. Although 20032004 Size Korea Project implemented by the Ministry of Knowledge Economy included obese subjects in the national body measurements survey, application for the obesity judgment criteria is not standardized in the clothing science field until now. If the obesity judgment criteria are not fixed, the results and their application by the criteria are not reliable. Therefore, the purpose of the study is to suggest reliable sizing charts for obese women, based on the KS apparel sizing system using reliable sampling methods and obese judgment criteria for obesity. Before the analyzing body measurement data, 499 female adults classified as "obese" in 5 out of 7 frequent used obesity indices were selected according to the previous study (Yi, 2009).

\section{Methods}

\section{The Subjects}

The subjects in the study were 499 females aged from the twenties to fifties. The body measurement data were originally from the 2003-2004 Size Korea Project (Table 1). 7 obesity judgment indices (Röhrer index $\geq 1.6$, Vervaeck index $\geq 94.7$, BMI $\geq 25$, Relative weight $\geq 120$, WHR $\geq 0.85$, Bust girth $\geq 91$ and Waist girth $\geq 78.5$ ) were examined and selected through previous studies of obesity-related fields, the subjects satisfying the obese condition in 5 obesity judgment indices out of these 7 indices were targeted in this study.

In distribution of subjects ranged from the twenties to fifties, the percent of the fifties is much higher than other age groups. The number of subjects in their fifties is remarkable. Conclusively, the portion of subjects over fifties is very significant compared to younger age groups. The ranks of these rates show the same as the as the 2007 report of Korean health and nutrition survey (NHIC, 2009).

\section{Statistics}

For the establishment of apparel sizing system, bust girth, waist girth, hip girth and height are used for statistics as key measurements. Crosstabulation table of bust girth and hip girth by height was given for making fitted outerwear covering the upper body such as jackets and shirts. In addition, crosstabulation

Table 1. Subjects by age groups

\begin{tabular}{c|c|c|c|c}
\hline \hline \multirow{2}{*}{ Age groups } & \multicolumn{2}{|c|}{ Size Korea } & \multicolumn{2}{c}{ This study } \\
\cline { 2 - 5 } & frequency & $\%$ & frequency & 81 \\
\hline The twenties & 692 & 31.0 & 116 & 23.0 \\
\hline The thirties & 735 & 33.0 & 104 & 21.0 \\
\hline The forties & 412 & 17.0 & 238 & 48.0 \\
\hline The fifties & 373 & 100.0 & 499 & 100.0 \\
\hline Total & 2,212 & & & \\
\hline
\end{tabular}


table of waist girth and hip girth was given. Size intervals for each sizing chart were compared 5 pitches with 3 pitches in each girth measurements. 3 pitches are generally used for people with normal weight in the KS Apparel sizing system. 5 pitches were introduced in the study due to higher usability and cost efficiency in the fashion industry.

Final suggestion of the sizing system for obese women, coverage and coverage efficiency were computed in crosstabulation table by observation percentages (over $1 \%$, over $2 \%$ and over $3 \%$ ). Size intervals and observation percentages indicating the highest coverage efficiency were suggested as final apparel sizing charts. Size designations for each fitted outerwear covering the upper body or lower body were introduced in this study by the body measurements of control dimension and referable dimension.

\section{Results}

\section{Body Size Analysis of the Subjects}

The comparative results of body measurements between the subjects of this study and entire group of females of 2003-2004 Size Korea were given to <Table $2>$. First of all, the subjects of this study were smaller than the entire female adults in all height measurements. To the contrary, the subjects of this study were much larger in all width measurements and depth measurements. In the case of lengths, there were different results according to the measurements. Namely, subjects of this study were smaller than entire group of females in the measurements such as Vertical Trunk Length, Shoulder Length, Total Length, Upperarm Length, Arm Length, Underarm Length, Cervicale to Wrist Length, Waist to Hip Length, Outside Leg Length. These measurements had positive and significant relationship with obesity in previous studies. From the study, Waist Front and Back Length, interscye-related lengths, Scye Depth, Trunk Circumference, Crotch Lengths were much higher than the result of 2003-2004 Size Korea.

All circumference measurements and Weight were much larger than those of 2003-2004 Size Korea. The average age of subjects in the study was older than that of Size Korea. It is concluded that obesity is a sign of getting older. According to the analysis of the drop values (Hip Circumference-Bust Circumference, Hip Circumference-Waist Circumference), it could be summarized that subjects were much bulkier and boxy in shape (namely, $\mathrm{H}$ shape) than normal people. The subjects were definitely more obese than the average of entire women. Waist/Height and WHR showed big differences between the subjects and the entire women.

\section{Crosstabulation Analysis for Establishing Sizing System}

\section{1) Sizing System for Outerwear Covering the Upper Body}

Before the suggestion of apparel sizing system for obese women, frequently used size intervals were reviewed and surveyed through previous studies (Ha, 2008, 2009; Kim, 2007a) and market research. Height, bust girth and hip girth were investigated not only in the KS apparel sizing system but also markets as size designation or control dimension. Many of size codes were not numeric codes utilizing body measurements but simple codes such as 88, 99, 100 etc. Nonetheless, most of them indicated the body measurements of control dimensions on the size labels. Lots of apparel brands didn't use even intervals in their size specification. Even though many of the brands use $3 \mathrm{~cm}$ pitches as a size interval, they don't use the same interval in bigger sizes. Most brands use $5 \mathrm{~cm}$ pitches in bigger size range, while they use $3 \mathrm{~cm}$ pitches in normal sized range. Therefore, $5 \mathrm{~cm}$ pitches and $3 \mathrm{~cm}$ pitches were used for selecting optimal size charts in the study. Based on the KS apparel sizing system, height, bust girth and hip girth were chosen as control dimensions for fitted outerwear covering the upper body. In the case of size interval, height was used in $5 \mathrm{~cm}$ pitches, bust girth and hip girth were divided in $5 \mathrm{~cm}$ and $3 \mathrm{~cm}$ pitches respectively.

First, bust girth and hip girth's pitches were set to $3 \mathrm{~cm}$, height's pitches were set to $5 \mathrm{~cm}$ in the size code of "bust girth - hip girth - height".

Crosstabulation table of bust girth and hip girth by height was presented in <Table $3>$. Over $1 \%$ of the observed cells were highlighted in $\langle$ Table 3$\rangle .5 \mathrm{~cm}$ 
Table 2. Descriptive analysis of the subjects

\begin{tabular}{|c|c|c|c|c|c|c|c|c|c|}
\hline \multirow[t]{2}{*}{ Measurements } & \multicolumn{2}{|c|}{$\begin{array}{l}\text { This study } \\
(\mathrm{n}=499)\end{array}$} & \multicolumn{2}{|c|}{\begin{tabular}{|c|} 
Size Korea \\
Total $(\mathrm{n}=2,212)$
\end{tabular}} & \multirow[t]{2}{*}{ Measurements } & \multicolumn{2}{|c|}{$\begin{array}{l}\text { This study } \\
(\mathrm{n}=499)\end{array}$} & \multicolumn{2}{|c|}{$\begin{array}{c}\text { Size Korea Total } \\
(\mathrm{n}=2,212)\end{array}$} \\
\hline & Mean & SD & Mean & SD & & Mean & SD & Mean & SD \\
\hline Stature & 154.35 & 5.82 & 157.54 & 5.40 & Upperarm Length & 30.44 & 1.53 & 30.57 & 1.40 \\
\hline Cervical Height & 131.46 & 5.48 & 134.03 & 4.93 & Arm Length & 52.89 & 2.28 & 52.83 & 2.31 \\
\hline Acromion Height & 124.82 & 5.22 & 127.17 & 4.73 & Underarm Length & 42.25 & 2.48 & 42.77 & 2.44 \\
\hline Shoulder Height & 125.95 & 5.23 & 128.31 & 4.71 & Cervicale to Wrist Length & 74.67 & 3.54 & 74.87 & 3.45 \\
\hline Axilla Height & 114.37 & 4.95 & 117.25 & 4.64 & Waist to Hip Length & 22.31 & 3.18 & 22.60 & 2.67 \\
\hline Hip Height & 74.15 & 3.98 & 76.11 & 3.72 & Outside Leg Length & 95.68 & 4.34 & 98.42 & 4.40 \\
\hline Crotch Height & 68.00 & 3.53 & 70.39 & 3.61 & Trunk Circumference & 155.07 & 6.65 & 150.15 & 6.47 \\
\hline Waist Height & 94.68 & 4.41 & 97.47 & 4.29 & $\begin{array}{l}\text { Crotch Length } \\
\text { (Natural Indentation) }\end{array}$ & 75.63 & 4.81 & 73.17 & 4.47 \\
\hline Waist Height (Omphalion) & 88.63 & 4.33 & 91.61 & 4.24 & Crotch Length (Omphalion) & 63.58 & 4.17 & 61.59 & 4.00 \\
\hline Biacromial Breadth & 36.41 & 1.74 & 36.00 & 1.70 & Neck Base Circumference & 39.14 & 2.01 & 37.43 & 2.22 \\
\hline Chest Breadth & 29.67 & 1.88 & 28.10 & 1.87 & Chest Circumference & 92.82 & 4.80 & 85.78 & 5.91 \\
\hline Bust Breadth & 30.77 & 2.22 & 28.24 & 2.26 & Bust Circumference & 96.96 & 5.87 & 86.89 & 7.76 \\
\hline Waist Breadth & 28.81 & 2.31 & 25.51 & 2.67 & Underbust Circumference & 83.62 & 4.78 & 75.85 & 6.09 \\
\hline Waist Breadth (Omphalion) & 31.58 & 2.39 & 28.54 & 2.77 & $\begin{array}{l}\text { Waist Circumference } \\
\text { (Natural Indentation) }\end{array}$ & 86.27 & 6.44 & 73.92 & 8.83 \\
\hline Hip Breadth & 33.47 & 1.66 & 32.57 & 1.66 & $\begin{array}{l}\text { Waist Circumference } \\
\text { (Omphalion) }\end{array}$ & 91.02 & 6.61 & 79.82 & 8.70 \\
\hline Chest Depth & 21.14 & 1.73 & 19.10 & 1.93 & Hip Circumference & 96.52 & 4.95 & 92.00 & 5.14 \\
\hline Bust Depth & 25.61 & 1.96 & 22.30 & 2.64 & Thigh Circumference & 57.68 & 4.00 & 54.35 & 4.16 \\
\hline Waist Depth & 23.88 & 2.23 & 19.71 & 3.08 & Midthigh Circumference & 50.38 & 3.73 & 47.34 & 3.84 \\
\hline Waist Depth (Omphalion) & 23.69 & 2.48 & 19.88 & 2.92 & Knee Circumference & 35.92 & 2.34 & 34.50 & 2.27 \\
\hline Hip Depth & 23.87 & 2.27 & 21.69 & 2.19 & Lower Knee Circumference & 33.20 & 2.28 & 32.00 & 2.07 \\
\hline Body Rise & 26.69 & 2.34 & 27.08 & 2.07 & Calf Circumference & 35.88 & 2.66 & 34.14 & 2.42 \\
\hline Vertical Trunk Length & 63.46 & 3.02 & 63.64 & 2.57 & Minimum Leg Circumference & 21.53 & 1.32 & 20.71 & 1.29 \\
\hline Waist Front Length & 33.97 & 2.55 & 32.78 & 2.20 & Ankle Circumference & 24.17 & 1.40 & 23.49 & 1.25 \\
\hline $\begin{array}{l}\text { Waist Front Length } \\
\text { (Omphalion) }\end{array}$ & 40.22 & 2.71 & 38.76 & 2.30 & Armscye Circumference & 41.69 & 2.99 & 38.13 & 3.37 \\
\hline Interscye, front & 33.34 & 1.75 & 32.50 & 1.87 & Upper Arm Circumference & 30.45 & 2.22 & 27.26 & 2.82 \\
\hline Interscye Fold, front & 34.02 & 2.23 & 32.25 & 2.36 & Elbow Circumference & 27.59 & 2.06 & 25.43 & 2.18 \\
\hline Bust Point-Bust Point & 19.22 & 1.84 & 17.74 & 1.93 & Wrist Circumference & 15.88 & .82 & 15.05 & .91 \\
\hline Shoulder Length & 12.64 & 1.00 & 12.66 & 1.08 & Weight & 64.86 & 7.95 & 56.44 & $\overline{7.79}$ \\
\hline Scye Depth & 17.62 & 1.52 & 17.21 & 1.58 & Age & 45.58 & 10.13 & 36.43 & 11.01 \\
\hline $\begin{array}{l}\text { Waist Back Length } \\
\text { (Natural Indentation) }\end{array}$ & 39.04 & 2.85 & 38.50 & 2.29 & $\begin{array}{l}\text { Hip Circumference- } \\
\text { Bust Circumference }\end{array}$ & -0.44 & 5.08 & 5.11 & 5.70 \\
\hline $\begin{array}{l}\text { Waist Back Length } \\
\text { (Omphalion) }\end{array}$ & 44.90 & 2.83 & 44.21 & 2.31 & $\begin{array}{l}\text { Hip Circumference- } \\
\text { Waist Circumference }\end{array}$ & 10.25 & 5.95 & 18.08 & 6.61 \\
\hline Total length & 134.35 & 5.68 & 136.47 & 4.98 & BMI & 27.15 & 2.29 & 22.77 & 3.17 \\
\hline Biacromion Length & 40.20 & 2.42 & 39.66 & 2.29 & Rohrer Index & 1.76 & .15 & 1.45 & .22 \\
\hline Interscye, back & 38.07 & 2.41 & 36.90 & 2.30 & Vervaeck Index & 104.79 & 6.71 & 91.07 & 9.84 \\
\hline Interscye Fold, back & 37.50 & 3.17 & 35.55 & 2.76 & Relative Weight & 132.84 & 11.31 & 109.65 & 16.37 \\
\hline Neck Point to Breast Point & 28.74 & 2.39 & 26.47 & 2.51 & Waist/Height & .56 & .04 & .47 & .06 \\
\hline $\begin{array}{l}\text { Neck Point to Breast Point to } \\
\text { Waistline }\end{array}$ & 42.15 & 2.60 & 40.64 & 2.39 & WHR (Waist/Hip) & .89 & .06 & .80 & .07 \\
\hline
\end{tabular}


Table 3. Crosstabulation table of bust girth \& hip girth by height - in $5 \mathrm{~cm}$ pitches

\begin{tabular}{|c|c|c|c|c|c|c|c|c|c|c|c|}
\hline Height & Hip Bust & 85 & 90 & 95 & 100 & 105 & 110 & 115 & 120 & 125 & Total \\
\hline \multirow{2}{*}{135} & 100 & & & & & $1(0.2)$ & & & & & $1(\quad 0.2)$ \\
\hline & Sub Total & & & & & $1(0.2)$ & & & & & $1(\quad 0.2)$ \\
\hline \multirow{2}{*}{140} & 100 & & & & & $1(0.2)$ & & & & & $1(\quad 0.2)$ \\
\hline & Sub Total & & & & & $1(0.2)$ & & & & & $1(\quad 0.2)$ \\
\hline \multirow{6}{*}{145} & 85 & & & & $1(0.2)$ & & & & & & $1(\quad 0.2)$ \\
\hline & 90 & & $41(8.2)$ & $5(1.0)$ & & $1(0.2)$ & & & & & $47(9.4)$ \\
\hline & 95 & & $3(0.6)$ & $6(1.2)$ & $2(0.4)$ & & & & & & $11(\quad 2.2)$ \\
\hline & 100 & & & $1(0.2)$ & & $1(0.2)$ & $1(0.2)$ & & & & $3(\quad 0.6)$ \\
\hline & 105 & & & & & & $1(0.2)$ & & & & $1\left(\begin{array}{ll}0.2\end{array}\right)$ \\
\hline & Sub Total & & $44(8.8)$ & $12(2.4)$ & $3(0.9)$ & $2(0.4)$ & $2(0.4)$ & & & & $63(12.6)$ \\
\hline \multirow{7}{*}{150} & 85 & & $2(0.4)$ & $3(0.6)$ & & & & & & & $5\left(\begin{array}{ll}1.0 \\
\end{array}\right.$ \\
\hline & 90 & $1(0.2)$ & $15(3.0)$ & $18(3.6)$ & $5(1.0)$ & $1(0.2)$ & & & & & $40(\quad 8.0)$ \\
\hline & 95 & & $6(1.2)$ & 19 ( 3.8) & $7(1.4)$ & $4(0.8)$ & & & & & $36(7.2)$ \\
\hline & 100 & & $5(1.0)$ & $5(1.0)$ & $9(1.8)$ & $7(1.4)$ & $3(0.6)$ & & & & $29(5.8)$ \\
\hline & 105 & & & $1(0.2)$ & $1(0.2)$ & $2(0.4)$ & & $1(0.2)$ & & & $5\left(\begin{array}{ll}5(1.0) \\
\end{array}\right.$ \\
\hline & 110 & & & & & & $1(0.2)$ & & & & $1(\quad 0.2)$ \\
\hline & Sub Total & $1(0.2)$ & $28(5.6)$ & $46(3.2)$ & $22(4.4)$ & $14(2.8)$ & $4(0.8)$ & $1(0.2)$ & & & $116(23.2)$ \\
\hline \multirow{6}{*}{155} & 90 & & $3(0.6)$ & $6(1.2)$ & $5(1.0)$ & $3(0.6)$ & & & & & $17(3.4)$ \\
\hline & 95 & & $8(1.6)$ & $43(8.6)$ & 19 ( 3.8$)$ & $3(0.6)$ & $2(0.4)$ & & & & $75(15.0)$ \\
\hline & 100 & & $7(1.4)$ & $23(4.6)$ & $24(4.8)$ & $3(0.6)$ & $7(1.4)$ & & $1(0.2)$ & & $65(13.0)$ \\
\hline & 105 & & $1(0.2)$ & $1(0.2)$ & $3(0.6)$ & $4(0.8)$ & $1(0.2)$ & & & & $10(2.0)$ \\
\hline & 110 & & & & & $2(0.4)$ & & & & & $2(0.4)$ \\
\hline & Sub Total & & 19(3.8) & $73(14.6)$ & $51(10.2)$ & $15(3.0)$ & $10(2.0)$ & & $1(0.2)$ & & $169(33.8)$ \\
\hline \multirow{6}{*}{160} & 90 & & & $1(0.2)$ & & & & & & & $1(\quad 0.3)$ \\
\hline & 95 & & $7(1.4)$ & $21(4.2)$ & $14(2.8)$ & $4(0.8)$ & & & & & $46(\quad 9.2)$ \\
\hline & 100 & & $9(1.8)$ & $17(3.4)$ & $11(2.2)$ & 5( 1.0) & $2(0.4)$ & $1(0.2)$ & & & $445(9.0)$ \\
\hline & 105 & & & $2(0.4)$ & 7( 1.4) & $4(0.8)$ & $5(1.0)$ & & & & $18(3.6)$ \\
\hline & 110 & & $1(0.2)$ & & & $1(0.2)$ & $1(0.2)$ & & & & $3(\quad 0.6)$ \\
\hline & Sub Total & & 17 ( 3.4) & $41(8.2)$ & $32(6.4)$ & $14(2.8)$ & $8(1.6)$ & $1(0.2)$ & & & $113(22.6)$ \\
\hline \multirow{4}{*}{165} & 95 & & & & $4(0.8)$ & $2(0.4)$ & & & & & $6\left(\begin{array}{ll}1.2 \\
6\end{array}\right.$ \\
\hline & 100 & & & $5(1.0)$ & $7(1.4)$ & $1(0.2)$ & & & & & $13(2.6)$ \\
\hline & 105 & & $1(0.2)$ & $5(1.0)$ & $2(0.4)$ & $3(0.6)$ & & & & $1(0.2)$ & $12(\quad 2.4)$ \\
\hline & Sub Total & & $1(0.2)$ & $11(2.2)$ & $13(2.6)$ & $6(1.2)$ & & & & $1(0.2)$ & $31(6.2)$ \\
\hline \multirow{4}{*}{170} & 100 & & & $1(0.2)$ & $1(0.2)$ & & & & & & $2(\quad 0.4)$ \\
\hline & 110 & & & & & $1(0.2)$ & & & & & $\begin{array}{ll}1( & 0.2)\end{array}$ \\
\hline & 120 & & & & & & & $1(0.2)$ & & & $1(\quad 0.2)$ \\
\hline & Sub Total & & & $1(0.2)$ & $1(0.2)$ & $1(0.2)$ & & $1(0.2)$ & & & $4\left(\begin{array}{ll}0.8 \\
)\end{array}\right.$ \\
\hline \multirow{2}{*}{175} & 110 & & & & & & & $1(0.2)$ & & & $1(\quad 0.2)$ \\
\hline & \begin{tabular}{|l|} 
Sub Total \\
\end{tabular} & & & & & & & $1(0.2)$ & & & $1(\quad 0.2)$ \\
\hline \multicolumn{2}{|r|}{ Total } & $1(0.2)$ & $109(21.8)$ & $183(36.7)$ & $122(24.4)$ & $54(10.8)$ & $24(4.8)$ & $4(0.8)$ & $1(0.2)$ & $1(0.2)$ & $499(100.0)$ \\
\hline
\end{tabular}


pitches in each girth measurement and height were adopted, based on the market survey as mentioned above.

When height ranged from 135 to 175 , the number of intervals was 9. With Bust girth ranged from 85 to 125 and the number of intervals was 9 . When hip girth ranged from 85 to 120 , the number of intervals was 8 .

The cell with the highest observation rate was 95 95-155, the value was $8.6 \%$.

The second highest was 100-100-155 with $4.8 \%$. Next, they appeared in the order of 95-100-155 (4.6\%) and 95-95-160 (4.2\%).

Highest observed cells ranked in the order of 8590-155 (5.39\%), 80-90-160 (4.44\%) and 80-90-155 $(4.31 \%)$ in $\mathrm{KS} \mathrm{K} 0051$. When these results were compared to KS apparel size, distinct body characteristics of obese women were reflected in this size chart.

<Table 4> showed a crosstabulation table of bust and hip girth by height.

$3 \mathrm{~cm}$ pitches in each girth measurement were adopted and $5 \mathrm{~cm}$ pitches in height, based on the KS apparel size, because this was suggested for fitted outerwear covering the upper body.

Over $1 \%$ of the observed cells were also highlighted in $\langle$ Table 4$\rangle$. $3 \mathrm{~cm}$ pitches in each girth measurement were adopted, based on the market survey as mentioned above. In the case of height, the pitches were set to $5 \mathrm{~cm}$ according to the result of the market research.

Height ranged from 135 to 175 , the number of intervals was 9. Bust girth ranged from 88 to 124 , the number of intervals was 12 and hip girth ranged from 88 to 124 , the number of intervals was 13 .

The cell with the highest observation rate was 88 $88-145$, the percentage was $7.4 \%$. Next in order was 94-97-155, the percentage was $3.8 \%$.

Next, they appeared in the order of 97-94-155 (2.8\%), 97-97-160 (2.8\%) and 94-94-155 (2.6\%).

On the other hand, highest observed cells appeared in the order of 85-88-155 (2.20\%), 82-88-155 (1.94\%), 91-91-155 (1.94\%) and 85-91-155 (1.77\%) in KS K 0051. Conclusively, suggested size chart showed distinct body characteristics of obese women compared to KS apparel size standard.

\section{2) Crosstabulation Analysis for Outerwear Cov- ering the Lower Body}

Based on the KS apparel sizing system, waist girth and hip girth were chosen as control dimensions for fitted outerwear covering the lower body. In the case of size interval, $5 \mathrm{~cm}$ pitches and $3 \mathrm{~cm}$ pitches were also used for selecting optimal size charts in the study.

First, $5 \mathrm{~cm}$ pitched size chart was given in <Table $5>$. A size chart with $5 \mathrm{~cm}$ pitches was introduced by the market research of plus-sized women's wears in this study. With waist girth ranged from 75 to 115 , the number of intervals was 9 , when hip girth ranged from 85 to 120 , the number of intervals was 7 . The cells with over $1 \%$ of the observation rate were also highlighted in <Table 5>.

The highest observed cell was $85-95$, and $12.8 \%$ of the entire user was distributed. The second highest was $85-90$ interval with $12.2 \%$, the third highest was 80-95 interval, with the observation rate of $11.8 \%$. Next, they appeared in the order of 85-100 (9.2\%), 90-100 (7.4\%) and 80-100 (6.6\%).

$3 \mathrm{~cm}$ pitches in waist and hip girth measurement were also adopted by the KS Apparel Sizing System as mentioned above.

The result of $3 \mathrm{~cm}$ pitched size chart for garments covering the lower body was in <Table 6>. In the case of $3 \mathrm{~cm}$ pitched size chart, waist girth and hip girth were also selected as control dimensions of the outerwear covering the lower body based on the KS Apparel Sizing System. With waist girth ranged from 73 to 115 , the number of intervals was 15 . When hip girth ranged from 85 to 121 , the number of intervals was 11 . The cell with the highest observation rate was $82-88$, the percentage was $8.4 \%$. Next in order was $82-97$ with $6.2 \%$ and $82-100$ with the percentage of $4.6 \%$ each. Next, they appeared in the order of 7994 (4.4\%), 85-97 (4.4\%), 85-94 (3.8\%).

Highest observed cells ranked in the order of 6791 (5.17\%), 64-88 (5.04\%) and 73-91 (4.27\%) in KS $\mathrm{K} 0051$. When these results were compared to KS apparel size, body characteristics of obese women were reflected distinctively in this size chart. 
Table 4. Crosstabulation table of bust girth \& hip girth by height - in $3 \mathrm{~cm}$ pitches

\begin{tabular}{|c|c|c|c|c|c|c|c|c|c|c|c|c|c|c|}
\hline Height & ${ }_{H} B$ & 88 & 91 & 94 & 97 & 100 & 103 & 106 & 109 & 112 & 115 & 121 & 124 & Total \\
\hline \multirow{2}{*}{135} & 100 & & & & & & & $1(0.2)$ & & & & & & $1(\quad 0.2)$ \\
\hline & Ttl & & & & & & & $1(0.2)$ & & & & & & $1(0.2)$ \\
\hline \multirow{2}{*}{140} & 100 & & & & & & $1(0.2)$ & & & & & & & $1(\quad 0.2)$ \\
\hline & $\mathrm{Ttl}$ & $2(0.4)$ & & & $2(0.4)$ & 1(0.2) & 1( 0.2$)$ & & & & & & & $6(1.2)$ \\
\hline \multirow{7}{*}{145} & 88 & $37(7.4)$ & $1(0.2)$ & $1(0.2)$ & $1(0.2)$ & & $1(0.2)$ & & & & & & & $41(8.2)$ \\
\hline & 91 & $2(0.4)$ & $2(0.4)$ & $3(0.6)$ & & & & & & & & & & $7\left(\begin{array}{ll}1.4\end{array}\right)$ \\
\hline & 94 & $1(0.2)$ & $2(0.4)$ & $3(0.6)$ & $2(0.4)$ & & & & & & & & & $8(\quad 1.6)$ \\
\hline & 97 & & & $2(0.4)$ & $1(0.2)$ & & & & $1(0.2)$ & & & & & $3(0.6)$ \\
\hline & 100 & & & & $1(0.2)$ & & $1(0.2)$ & & $1(0.2)$ & & $1(0.2)$ & & & $3(\quad 0.6)$ \\
\hline & 103 & & & & & & & $1(0.2)$ & & $1(0.2)$ & & & & $1(\quad 0.2)$ \\
\hline & $\mathrm{Ttl}$ & $40(8.0)$ & $5(1.0)$ & $9(1.8)$ & $5(1.0)$ & & 2( 0.4$)$ & $1(0.2)$ & $2(0.4)$ & $1(0.2)$ & $1(0.2)$ & & & $63(12.6)$ \\
\hline \multirow{9}{*}{150} & 85 & & & $3(0.6)$ & & & & & & & & & & $3(\quad 0.5)$ \\
\hline & 88 & $1(0.2)$ & $6(1.2)$ & $8(1.6)$ & $3(0.6)$ & 1(0.2) & & & & & & & & $19(3.8)$ \\
\hline & 91 & $3(0.6)$ & $8(1.6)$ & $7(1.4)$ & $3(0.6)$ & $1(0.2)$ & 1( 0.2$)$ & & & & & & & $23(4.6)$ \\
\hline & 94 & $2(0.4)$ & 4( 0.8$)$ & $5(1.0)$ & $6(1.2)$ & 4( 0.8$)$ & 2( 0.4$)$ & $1(0.2)$ & & & & & & $24(4.8)$ \\
\hline & 97 & & $3(0.6)$ & $7(1.4)$ & $4\left(\begin{array}{ll}0.8) \\
\end{array}\right.$ & \begin{tabular}{|ll}
$2(0.4)$ \\
\end{tabular} & \begin{tabular}{|ll}
1( & $0.2)$
\end{tabular} & 1( 0.2$)$ & $1(0.2)$ & & & & & $19(3.8)$ \\
\hline & 100 & $1(0.2)$ & 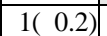 & $2(0.4)$ & $1(0.2)$ & $6(1.2)$ & $\begin{array}{|cc|}3( & 0.6) \\
\end{array}$ & & $1(0.2)$ & & & & & $15(3.0)$ \\
\hline & 103 & & & $1(0.2)$ & $1(0.2)$ & $1(0.2)$ & 4( 0.8$)$ & 4( 0.8$)$ & & & $1(0.2)$ & & & $12(\quad 2.4)$ \\
\hline & 109 & & & & & & & & & $1(0.2)$ & & & & $1(\quad 0.2)$ \\
\hline & Ttl & $7(1.4)$ & $22(4.4)$ & 33 ( 6.6) & $18(3.6)$ & $15(3.0)$ & $11(2.2)$ & $6(1.2)$ & $2(0.4)$ & $1(0.2)$ & $1(0.2)$ & & & $116(23.2)$ \\
\hline \multirow{9}{*}{155} & 88 & & & & & & $1(0.2)$ & & & & & & & $1(\quad 0.2)$ \\
\hline & 91 & & $3(0.6)$ & $3(0.6)$ & $4(0.8)$ & 4( 0.8$)$ & 2( 0.4$)$ & & & & & & & $16(3.2)$ \\
\hline & 94 & $1(0.2)$ & 3( 0.6$)$ & $13(2.6)$ & $14(2.8)$ & $10(2.0)$ & 1( 0.2$)$ & $1(0.2)$ & $2(0.4)$ & & & & & $44(8.8)$ \\
\hline & 97 & $1(0.2)$ & $7(1.4)$ & 19 ( 3.8) & $17(3.4)$ & $5(1.0)$ & 3( 0.6$)$ & $2(0.4)$ & $1(0.2)$ & & & & & $55(11.0)$ \\
\hline & 100 & & $2(0.4)$ & $7(1.4)$ & $6(1.2)$ & $12(2.4)$ & & $1(0.2)$ & $5(1.0)$ & & & & & $34(6.8)$ \\
\hline & 103 & & $2(0.4)$ & & $2(0.4)$ & $2(0.4)$ & $4(0.8)$ & $2(0.4)$ & $1(0.2)$ & & & $1(0.2)$ & & $14(2.8)$ \\
\hline & 106 & & & & $2(0.4)$ & & & 1( 0.2) & & & & & & $3(0.6)$ \\
\hline & 109 & & & & & & $1(0.2)$ & $1(0.2)$ & & & & & & $2(\quad 0.4)$ \\
\hline & Ttl & $2(0.4)$ & $17(3.4)$ & $42(8.4)$ & $45(9.0)$ & $33(6.6)$ & 12(2.4) & $8(1.6)$ & $9(1.8)$ & & & $1(0.2)$ & & $169(33.88)$ \\
\hline \multirow{8}{*}{160} & 91 & & $1(0.2)$ & $1(0.2)$ & & & & & & & & & & $2(0.4)$ \\
\hline & 94 & & $3(0.6)$ & $7(1.4)$ & $1(0.2)$ & $2(0.4)$ & $5(1.0)$ & & & & & & & $18(3.6)$ \\
\hline & 97 & $1(0.2)$ & 4( 0.8$)$ & $9(1.8)$ & $14(2.8)$ & 8( 1.6) & 1(0.2) & $1(0.2)$ & & & & & & $38(7.6)$ \\
\hline & 100 & & $5(1.0)$ & $7(1.4)$ & $6(1.2)$ & $3(0.6)$ & 3( 0.6$)$ & $2(0.4)$ & $1(0.2)$ & $1(0.2)$ & & & & $28(5.66)$ \\
\hline & 103 & & $2(0.4)$ & & $3(0.6)$ & \begin{tabular}{|l|}
$7(1.4)$ \\
\end{tabular} & $2(0.4)$ & 4( 0.8$)$ & $1(0.2)$ & & $1(0.2)$ & & & $20(4.0)$ \\
\hline & 106 & & & & $1(0.2)$ & & & $1(0.2)$ & $3(0.6)$ & & & & & $5(1.0)$ \\
\hline & 109 & & $1(0.2)$ & & & & & & $1(0.2)$ & & & & & $2(0.4)$ \\
\hline & Ttl & $1(0.2)$ & $16(3.2)$ & $24(4.8)$ & $25(5.0)$ & $20(4.0)$ & 11( 2.2) & $8(1.6)$ & $6(1.2)$ & 1( 0.2$)$ & $1(0.2)$ & & & $113(22.6)$ \\
\hline \multirow{6}{*}{165} & 94 & & & & & $2(0.4)$ & & & & & & & & $2(\quad 0.4)$ \\
\hline & 97 & & & $1(0.2)$ & $1(0.2)$ & $2(0.4)$ & 3( 0.6) & $1(0.2)$ & & & & & & $8(\quad 1.6)$ \\
\hline & 100 & & & $2(0.4)$ & $2(0.4)$ & \begin{tabular}{|l|}
$3(0.6)$ \\
\end{tabular} & & & & & & & & $7(\quad 1.4)$ \\
\hline & 103 & & $1(0.2)$ & $2(0.4)$ & $1(0.2)$ & 3( 0.6$)$ & $1(0.2)$ & $1(0.2)$ & & & & & & $9(1.8)$ \\
\hline & 106 & & & $2(0.4)$ & & & $2(0.4)$ & & & & & & $1(0.2)$ & $5\left(\begin{array}{ll}1.0) \\
\end{array}\right.$ \\
\hline & Ttl & & $1(0.2)$ & $7(1.4)$ & $4(0.8)$ & $10(2.0)$ & $6(1.2)$ & $2(0.4)$ & & & & & $1(0.2)$ & $31(6.2)$ \\
\hline \multirow{4}{*}{170} & 100 & & & $1(0.2)$ & & $1(0.2)$ & & & & & & & & $2(\quad 0.4)$ \\
\hline & 109 & & & & & & $1(0.2)$ & & & & & & & $1(\quad 0.2)$ \\
\hline & 121 & & & & & & & & & & $1(0.2)$ & & & $1(\quad 0.2)$ \\
\hline & $\mathrm{Ttl}$ & & & $1(0.2)$ & & $1(0.2)$ & $1(0.2)$ & & & & $1(0.2)$ & & & $4(\quad 0.8)$ \\
\hline \multirow{2}{*}{175} & 109 & & & & & & & & & $1(0.2)$ & & & & $1(\quad 0.2)$ \\
\hline & $\mathrm{Ttl}$ & & & & & & & & & $1(0.2)$ & & & & $1(\quad 0.2)$ \\
\hline \multicolumn{2}{|c|}{ Total } & $50(10.0)$ & $61(12.2)$ & $116(23.2)$ & 97(19.4) & $79(15.8)$ & $44(8.8)$ & $26(5.2) 1$ & $18(3.6)$ & $3(0.6)$ & $3(0.6)$ & 1( 0.2$)$ & $1(0.2)$ & $499(100.0)$ \\
\hline
\end{tabular}


Table 5. Crosstabulation of waist girth by hip girth - in $5 \mathrm{~cm}$ pitches

\begin{tabular}{c|c|c|c|c|c|c|c|c|c|c}
\hline \multicolumn{1}{|c|}{ Waist } & \multirow{2}{*}{75} & 80 & 85 & 90 & 95 & 100 & 105 & 110 & 115 & Total \\
\hline 85 & & $2(0.4)$ & $4(0.8)$ & & & & & & & $6(1.2)$ \\
\hline 90 & $4(0.8)$ & $28(5.6)$ & $59(11.8)$ & $10(2.0)$ & $4(0.8)$ & & & & & $105(21.0)$ \\
\hline 95 & $2(0.2)$ & $61(12.2)$ & $64(12.8)$ & $34(6.8)$ & $11(2.2)$ & $2(0.4)$ & & & & $174(34.8)$ \\
\hline 100 & $6(1.2)$ & $33(6.6)$ & $46(9.2)$ & $37(7.4)$ & $20(4.0)$ & $14(2.8)$ & $2(0.4)$ & $1(0.2)$ & & $159(31.8)$ \\
\hline 105 & & $7(1.4)$ & $6(1.2)$ & $12(2.4)$ & $11(2.2)$ & $7(1.4)$ & $2(0.4)$ & & $1(0.2)$ & $52(9.2)$ \\
\hline 110 & & & $1(0.2)$ & $1(0.2)$ & $3(0.6)$ & $1(0.2)$ & $2(0.4)$ & & & $8(1.6)$ \\
\hline 120 & & & & & & & & & $1(0.2)$ & $1(0.2)$ \\
\hline Total & $12(2.4)$ & $131(26.2)$ & $180(36.0)$ & $94(18.8)$ & $49(9.8)$ & $24(4.8)$ & $6(1.2)$ & $1(0.2)$ & $2(0.4)$ & $499(100.0)$ \\
\hline
\end{tabular}

observed over $1 \%$

: observed over $2 \%$

observed over $3 \%$

Table 6. Crosstabulation table of waist girth by hip girth - in $3 \mathrm{~cm}$ pitches

\begin{tabular}{|c|c|c|c|c|c|c|c|c|c|c|c|c|c|c|c|c|}
\hline Hip & 73 & 76 & 79 & 82 & 85 & 88 & 91 & 94 & 97 & 100 & 103 & 106 & 109 & 112 & 115 & Total \\
\hline 85 & & & & $\begin{array}{c}1 \\
(0.2)\end{array}$ & $\begin{array}{c}2 \\
(0.4)\end{array}$ & & & & & & & & & & & $\begin{array}{l}3 \\
(0.6)\end{array}$ \\
\hline 88 & & $\begin{array}{c}1 \\
(0.2)\end{array}$ & $\begin{array}{c}8 \\
(1.6)\end{array}$ & $\begin{array}{c}42 \\
(8.4)\end{array}$ & $\begin{array}{c}4 \\
(0.8)\end{array}$ & $\begin{array}{l}6 \\
(1.2)\end{array}$ & & & & & & & & & & $\begin{array}{l}61 \\
(12.2)\end{array}$ \\
\hline 91 & & $\begin{array}{c}3 \\
(0.6)\end{array}$ & $\begin{array}{l}11 \\
(2.2)\end{array}$ & $\begin{array}{c}14 \\
(2.8)\end{array}$ & $\begin{array}{c}7 \\
(1.4)\end{array}$ & $\begin{array}{l}5 \\
(1.0)\end{array}$ & $\begin{array}{l}5 \\
(1.0)\end{array}$ & $\begin{array}{c}3 \\
(0.6)\end{array}$ & & & & & & & & $\begin{array}{c}48 \\
(\quad 9.6)\end{array}$ \\
\hline 94 & & & $\begin{array}{c}22 \\
(4.4)\end{array}$ & $\begin{array}{c}21 \\
(4.1)\end{array}$ & $\begin{array}{c}19 \\
(3.8)\end{array}$ & $\begin{array}{c}17 \\
(3.4)\end{array}$ & $\begin{array}{c}9 \\
(1.8)\end{array}$ & $\begin{array}{c}6 \\
(1.2)\end{array}$ & $\begin{array}{c}1 \\
(0.2)\end{array}$ & $\begin{array}{c}1 \\
(0.2)\end{array}$ & & & & & & $\begin{array}{c}96 \\
(19.2)\end{array}$ \\
\hline 97 & $\begin{array}{c}1 \\
(0.2)\end{array}$ & $\begin{array}{c}7 \\
(1.4)\end{array}$ & $\begin{array}{c}17 \\
(3.4)\end{array}$ & $\begin{array}{c}31 \\
(6.2)\end{array}$ & $\begin{array}{c}22 \\
(4.4)\end{array}$ & $\begin{array}{c}16 \\
(3.2)\end{array}$ & $\begin{array}{c}17 \\
(3.4)\end{array}$ & $\begin{array}{c}8 \\
(1.6)\end{array}$ & $\begin{array}{c}1 \\
(0.2)\end{array}$ & $\begin{array}{c}3 \\
(0.6)\end{array}$ & & & & & & $\begin{array}{l}123 \\
(24.6)\end{array}$ \\
\hline 100 & $\begin{array}{c}1 \\
(0.2)\end{array}$ & $\begin{array}{c}3 \\
(0.6)\end{array}$ & $\begin{array}{c}6 \\
(1.2)\end{array}$ & $\begin{array}{c}23 \\
(4.6)\end{array}$ & $\begin{array}{l}15 \\
(3.0)\end{array}$ & $\begin{array}{c}17 \\
(3.4)\end{array}$ & $\begin{array}{c}9 \\
(1.8)\end{array}$ & $\begin{array}{c}4 \\
(0.8)\end{array}$ & $\begin{array}{c}10 \\
(2.0)\end{array}$ & $\begin{array}{c}3 \\
(0.6)\end{array}$ & & & & & & $\begin{array}{l}91 \\
(18.2)\end{array}$ \\
\hline 103 & & $\begin{array}{c}1 \\
(0.2)\end{array}$ & & $\begin{array}{c}7 \\
(1.4)\end{array}$ & $\begin{array}{c}7 \\
(1.4) \\
\end{array}$ & $\begin{array}{c}7 \\
(1.4)\end{array}$ & $\begin{array}{c}8 \\
(1.6) \\
\end{array}$ & $\begin{array}{c}4 \\
(0.8)\end{array}$ & $\begin{array}{c}10 \\
(2.0)\end{array}$ & $\begin{array}{l}5 \\
(1.0) \\
\end{array}$ & $\begin{array}{l}5 \\
(1.0) \\
\end{array}$ & $\begin{array}{c}1 \\
(0.2)\end{array}$ & $\begin{array}{c}1 \\
(0.2)\end{array}$ & & & $\begin{array}{l}56 \\
(11.2)\end{array}$ \\
\hline 106 & & & $\begin{array}{c}1 \\
(0.2)\end{array}$ & $\begin{array}{c}1 \\
(0.2)\end{array}$ & & $\begin{array}{c}1 \\
(0.2)\end{array}$ & $\begin{array}{c}3 \\
(0.6)\end{array}$ & $\begin{array}{c}2 \\
(0.4)\end{array}$ & $\begin{array}{c}2 \\
(0.4)\end{array}$ & $\begin{array}{c}2 \\
(0.4)\end{array}$ & & & & & $\begin{array}{c}1 \\
(0.2)\end{array}$ & $\begin{array}{c}13 \\
(2.6)\end{array}$ \\
\hline 109 & & & & & $\begin{array}{c}1 \\
(0.2)\end{array}$ & & $\begin{array}{c}1 \\
(0.2)\end{array}$ & & $\begin{array}{c}3 \\
(0.6)\end{array}$ & & $\begin{array}{c}1 \\
(0.2)\end{array}$ & & & & & $\begin{array}{l}7 \\
(1.4)\end{array}$ \\
\hline 121 & & & & & & & & & & & & & & $\begin{array}{c}1 \\
(0.2)\end{array}$ & & $\begin{array}{l}1 \\
(0.2)\end{array}$ \\
\hline Total & $\begin{array}{c}2 \\
(0.4)\end{array}$ & $\begin{array}{l}15 \\
(3.0)\end{array}$ & $\begin{array}{c}65 \\
(13.0)\end{array}$ & $\begin{array}{c}140 \\
(28.0)\end{array}$ & $\begin{array}{c}77 \\
(14.4)\end{array}$ & $\begin{array}{c}69 \\
(13.8)\end{array}$ & $\begin{array}{c}52 \\
(10.4)\end{array}$ & $\begin{array}{c}27 \\
(5.4)\end{array}$ & $\begin{array}{c}27 \\
(5.4)\end{array}$ & $\begin{array}{l}14 \\
(2.8)\end{array}$ & $\begin{array}{c}6 \\
(1.2)\end{array}$ & $\begin{array}{c}1 \\
(0.2)\end{array}$ & $\begin{array}{c}1 \\
(0.2)\end{array}$ & $\begin{array}{c}1 \\
(0.2)\end{array}$ & $\begin{array}{c}1 \\
(0.2)\end{array}$ & $\begin{array}{c}499 \\
(100.0)\end{array}$ \\
\hline
\end{tabular}

3. Comparison of Coverage \& Coverage Efficiency in Each Size Charts

\section{1) Coverage \& Coverage Efficiency for the Out- erwear Covering the Upper Body}

Coverage means the coverage rate of selected spe- cific size intervals for total users' size distribution. According to previous studies on sizing systems, coverage rates over $1 \%, 2 \%$ and $3 \%$ of the observed cells were investigated in aspect of customer satisfaction of sizing systems and cost efficiency of product manufacturing. Customer satisfaction vs. cost efficiency 
is a contrary concept in manufacturing. In general, if lots of size numbers are provided, customer will be satisfied but manufacturer's cost will increase. Conclusively, offering optimal size numbers will be indispensable for meeting both requirements. The concept of coverage efficiency used in this study is a new concept considering the coverage of each size interval, it is computed by "coverage $\div$ the number of the observed intervals", namely, coverage per interval (Yi $\&$ Kim, 2008). If one's coverage efficiency were higher than others in size charts with same coverage rates, the side with higher coverage efficiency would satisfy customers. Therefore, the purpose of this study was to provide the size charts with the highest coverage efficiencies. Coverage \& coverage efficiency of outerwear covering the upper body was given in $\langle$ Table 7$\rangle$.

First of all, the number of cells with over $1 \%$ observation was 34 , with coverage $80.6 \%$ and coverage efficiency $2.37 \%$. The number of cells with over $2 \%$ observation was 12 , with coverage $53.0 \%$ and coverage efficiency $4.42 \%$. The number of cells with over $3 \%$ observation was 10 , with coverage $49.0 \%$ and coverage efficiency $4.9 \%$. Based on comparison of the coverage efficiencies, the highest coverage efficiency was confirmed by $5 \mathrm{~cm}$ size interval and observation rate of over $3 \%$.

\section{2) Coverage \& Coverage Efficiency for the Out- erwear Covering the Lower Body}

Coverage \& coverage efficiency of outerwear covering the lower body was in <Table $8>$. Waist girth and hip girth were chosen as control dimensions in outerwear covering the lower body. Each coverage and coverage efficiency of two pitches were examined by the observation rates, over $1 \%, 2 \%$ and $3 \%$.

The number of cells with over $1 \%$ observation rate was 17 , with coverage $92.0 \%$ and coverage efficiency $5.41 \%$. The number of cells with over $2 \%$ observation was 14 , with coverage $88 \%$ and coverage efficiency $6.29 \%$. The number of cells with over $3 \%$ observation was 9 , with coverage $76.4 \%$ and coverage efficiency $8.49 \%$. It is determined that the highest coverage efficiency was also in $5 \mathrm{~cm}$ size interval and observation rate of over $3 \%$.

Table 7. Coverage \& coverage efficiency of outerwear covering the upper body

\begin{tabular}{|c|c|c|c|c|}
\hline \multirow{2}{*}{$\begin{array}{l}\text { Control dimension } \\
\text { (Size pitch) }\end{array}$} & \multirow{2}{*}{ Remarks } & \multicolumn{3}{|c|}{ Observation } \\
\hline & & Over $1 \%$ & Over $2 \%$ & Over $3 \%$ \\
\hline \multirow{3}{*}{$\begin{array}{l}\text { Height }(5 \mathrm{~cm}) \\
\text { Bust Girth }(5 \mathrm{~cm}) \\
\text { Hip Girth }(5 \mathrm{~cm})\end{array}$} & No. of interval & 34 & 12 & 10 \\
\hline & Coverage (\%) & 80.6 & 53.0 & 49.0 \\
\hline & Coverage efficiency* & 2.37 & 4.42 & 4.90 \\
\hline \multirow{3}{*}{$\begin{array}{l}\text { Height }(5 \mathrm{~cm}) \\
\text { Bust Girth }(3 \mathrm{~cm}) \\
\text { Hip Girth }(3 \mathrm{~cm})\end{array}$} & No. of interval & 30 & 8 & 3 \\
\hline & Coverage $(\%)$ & 54.6 & 27.2 & 14.6 \\
\hline & Coverage efficiency* & 1.82 & 3.4 & 4.87 \\
\hline
\end{tabular}

$*$ coverage efficiency $=$ coverage $(\%) \div$ No. of intervals

Table 8. Coverage \& coverage efficiency of outerwear covering the lower body

\begin{tabular}{c|c|c|c|c}
\hline \hline \multirow{2}{*}{$\begin{array}{c}\text { Control dimension } \\
\text { (Size pitch) }\end{array}$} & Remarks & Over 1\% & Over 2\% & Over 3\% \\
\cline { 2 - 5 } & No. of interval & 17 & 14 & 9 \\
\hline \multirow{3}{*}{$\begin{array}{c}\text { Waist Girth }(5 \mathrm{~cm}) \\
\text { Hip Girth }(5 \mathrm{~cm})\end{array}$} & Coverage $(\%)$ & 92.0 & 88.0 & 76.4 \\
\cline { 2 - 5 } & Coverage efficiency* & 5.41 & 6.29 & 13 \\
\hline \multirow{3}{*}{$\begin{array}{c}\text { Waist Girth }(3 \mathrm{~cm}) \\
\text { Hip Girth }(3 \mathrm{~cm})\end{array}$} & No. of interval & 33 & 64.8 & 55.8 \\
\cline { 2 - 5 } & Coverage $(\%)$ & 86.6 & 3.81 & 4.29 \\
\hline
\end{tabular}

$*$ coverage efficiency $=$ coverage $(\%) \div$ No. of intervals 


\section{Suggestion for Apparel Sizing Charts for Obese Women}

\section{1) Sizing Charts for Outerwear Covering the Upper Body}

Coverage efficiencies according to pitches and observation rates were examined in $\langle$ Table 7$\rangle$. The optimal size pitches and observed intervals were selected. The size chart was provided by $5 \mathrm{~cm}$ pitches in all control dimensions and each size designation included the cells with over $3 \%$ observation rate. A total of 10 size codes were suggested in 〈Table 9>. 〈Table 9> shows the control dimensions and referable dimensions' body measurements that are necessary to make garments. These values were the average of subjects distributed in each cell. The values of bust girth, hip girth and height as control dimensions were given, 12 measurements as referable dimensions were provided.

\section{2) Sizing Charts for Outerwear Covering the Lower Body}

According to $\langle$ Table $8>$, the optimal size pitches

Table 9. Control \& referable dimensions dimensions by each size designation - for outerwear covering the upper body

\begin{tabular}{|c|c|c|c|c|c|c|c|c|c|c|c|c|c|c|c|}
\hline \multirow[b]{2}{*}{$\begin{array}{l}\text { Size } \\
\text { codes }\end{array}$} & \multicolumn{3}{|c|}{ Control dimensions } & \multicolumn{12}{|c|}{ Referable dimensions } \\
\hline & $\begin{array}{l}\text { Bust } \\
\text { Girth }\end{array}$ & $\begin{array}{l}\text { Hip } \\
\text { Girth }\end{array}$ & Height & $\begin{array}{l}\text { Waist } \\
\text { Girth }\end{array}$ & $\begin{array}{l}\text { Waist } \\
\text { Girth } \\
\text { (Omph- } \\
\text { alion) }\end{array}$ & $\begin{array}{l}\text { Waist } \\
\text { Back } \\
\text { Length }\end{array}$ & $\begin{array}{c}\text { Arm } \\
\text { Length }\end{array}$ & $\begin{array}{l}\text { Inter- } \\
\text { scye, } \\
\text { front }\end{array}$ & $\begin{array}{l}\text { Inter- } \\
\text { scye, } \\
\text { back }\end{array}$ & $\mid \begin{array}{c}\text { Biacro- } \\
\text { mion } \\
\text { Length }\end{array}$ & $\begin{array}{l}\text { Neck } \\
\text { Base } \\
\text { Girth }\end{array}$ & $\begin{array}{c}\text { Upper } \\
\text { Arm } \\
\text { Girth }\end{array}$ & \begin{tabular}{|c|} 
Neck \\
Point \\
to Breast \\
Point \\
to Waist- \\
line
\end{tabular} & $\begin{array}{c}\text { Bust } \\
\text { Point } \\
\text { to } \\
\text { Bust } \\
\text { Point }\end{array}$ & $\begin{array}{c}\text { Neck } \\
\text { Point } \\
\text { to } \\
\text { Breast } \\
\text { Point }\end{array}$ \\
\hline $90-90-145$ & 90 & 90 & 145 & 80.8 & 86.1 & 36.9 & 50.6 & 31.3 & 35.0 & 37.5 & 37.6 & 27.8 & 40.1 & 18.7 & 26.3 \\
\hline $90-90-150$ & 90 & 90 & 150 & 83.5 & 86.2 & 37.9 & 50.9 & 32.0 & 37.5 & 38.9 & 38.4 & 28.7 & 41.6 & 18.4 & 28.4 \\
\hline $95-90-150$ & 95 & 90 & 150 & 85.0 & 88.6 & 37.1 & 50.1 & 33.4 & 38.7 & 39.9 & 39.0 & 27.6 & 40.2 & 18.9 & 26.7 \\
\hline $95-95-150$ & 95 & 95 & 150 & 88.0 & 92.6 & 37.9 & 52.7 & 33.6 & 38.2 & 39.9 & 38.6 & 30.7 & 41.1 & 18.3 & 29.2 \\
\hline $95-95-155$ & 95 & 95 & 155 & 85.9 & 90.8 & 39.7 & 53.2 & 33.2 & 38.9 & 40.5 & 39.2 & 30.2 & 42.6 & 19.0 & 29.5 \\
\hline $95-100-155$ & 95 & 100 & 155 & 88.4 & 93.9 & 39.8 & 52.9 & 33.6 & 38.9 & 40.5 & 39.4 & 31.5 & 42.6 & 19.5 & 29.6 \\
\hline $100-95-155$ & 100 & 95 & 155 & 94.7 & 101.0 & 42.3 & 54.1 & 33.6 & 38.3 & 41.8 & 40.1 & 32.6 & 44.4 & 22.2 & 31.6 \\
\hline $100-100-155$ & 100 & 100 & 155 & 94.4 & 100.4 & 39.9 & 53.9 & 33.6 & 39.9 & 41.5 & 40.9 & 32.2 & 44.4 & 19.1 & 30.3 \\
\hline $95-95-160$ & 95 & 95 & 160 & 85.3 & 90.5 & 40.6 & 54.5 & 34.2 & 39.0 & 41.1 & 39.4 & 30.4 & 42.6 & 18.9 & 29.0 \\
\hline $95-100-160$ & 95 & 100 & 160 & 94.4 & 100.4 & 39.9 & 53.9 & 33.6 & 39.9 & 41.5 & 40.9 & 32.2 & 44.4 & 19.1 & 30.3 \\
\hline
\end{tabular}

Table 10. Control \& referable dimensions dimensions by each size designation - for outerwear covering the lower body

\begin{tabular}{|c|c|c|c|c|c|c|c|c|c|c|c|c|c|}
\hline \multirow[b]{2}{*}{$\begin{array}{l}\text { Size } \\
\text { codes }\end{array}$} & \multicolumn{2}{|c|}{$\begin{array}{c}\text { Control } \\
\text { dimensions }\end{array}$} & \multicolumn{11}{|c|}{ Referable dimensions } \\
\hline & $\begin{array}{l}\text { Waist } \\
\text { Girth }\end{array}$ & $\begin{array}{l}\text { Hip } \\
\text { Girth }\end{array}$ & $\begin{array}{l}\text { Waist } \\
\text { Girth } \\
\text { (Omph- } \\
\text { alion) }\end{array}$ & $\begin{array}{l}\text { Waist } \\
\text { to Hip } \\
\text { Length }\end{array}$ & $\begin{array}{l}\text { Crotch } \\
\text { Length }\end{array}$ & $\begin{array}{l}\text { Outside } \\
\text { Leg } \\
\text { Length }\end{array}$ & $\begin{array}{l}\text { Crotch } \\
\text { Height }\end{array}$ & $\begin{array}{l}\text { Waist } \\
\text { Height }\end{array}$ & $\begin{array}{l}\text { Body } \\
\text { Rise }\end{array}$ & $\begin{array}{l}\text { Thigh } \\
\text { Girth }\end{array}$ & $\begin{array}{l}\text { Knee } \\
\text { Girth }\end{array}$ & $\begin{array}{l}\text { Calf } \\
\text { Girth }\end{array}$ & $\begin{array}{c}\text { Minimum } \\
\text { Leg } \\
\text { Girth }\end{array}$ \\
\hline $80-90$ & 80 & 90 & 85.1 & 21.9 & 73.3 & 93.3 & 65.7 & 92.0 & 26.3 & 53.8 & 33.4 & 34.1 & 20.6 \\
\hline $80-95$ & 80 & 95 & 85.4 & 22.5 & 74.8 & 96.0 & 67.9 & 95.1 & 27.3 & 57.2 & 35.4 & 35.5 & 21.4 \\
\hline $80-100$ & 80 & 100 & 87.4 & 23.6 & 78.6 & 99.4 & 70.2 & 98.3 & 28.1 & 60.0 & 36.9 & 37.0 & 21.6 \\
\hline $85-90$ & 85 & 90 & 86.1 & 23.2 & 75.1 & 90.6 & 64.1 & 89.5 & 25.4 & 54.3 & 33.9 & 32.6 & 20.4 \\
\hline $85-95$ & 85 & 95 & 90.3 & 22.2 & 74.7 & 97.0 & 68.9 & 95.9 & 27.0 & 56.6 & 35.4 & 35.4 & 21.4 \\
\hline $85-100$ & 85 & 100 & 90.6 & 22.8 & 76.5 & 97.7 & 69.5 & 96.6 & 27.2 & 60.1 & 37.2 & 37.4 & 21.8 \\
\hline $90-95$ & 90 & 95 & 93.7 & 21.4 & 74.1 & 96.1 & 69.1 & 95.2 & 26.1 & 56.8 & 35.9 & 35.9 & 21.7 \\
\hline $90-100$ & 90 & 100 & 94.2 & 22.3 & 76.8 & 96.5 & 38.3 & 95.4 & 27.1 & 59.3 & 36.5 & 36.7 & 22.0 \\
\hline $95-100$ & 95 & 100 & 99.7 & 21.6 & 74.2 & 93.4 & 66.6 & 92.3 & 25.7 & 57.9 & 36.2 & 35.4 & 21.3 \\
\hline
\end{tabular}


and observation rate were selected. In $5 \mathrm{~cm}$ pitches and over $3 \%$ observation rate, coverage coefficient showed the highest value. Therefore, a size chart with $5 \mathrm{~cm}$ pitches was provided, which included the observed cells of over $3 \%$. Waist girth and hip girth were introduced as control dimensions, 11 referable dimensions were also given in $\langle$ Table 10〉. A total of 9 size codes was suggested for outwear covering the lower body.

\section{Conclusions}

This study was implemented to make suggestions for apparel size chart for obese women. Recently, it is remarkable to note the growing tendency of obesity in female adults in Korea. Nonetheless, proper size charts for obese women on the government level were not offered until now. To sample appropriate subjects, strict criteria for obesity judgment were used from previous studies, and a total of 499 female adults' body measurement data out of Size Korea's body measurement data were utilized in this study. The conclusion was as follows:

First, before the suggestion of apparel size charts for obese women, the body characteristics of 499 obese women were examined with the entire female group of Size Korea. In all heights and lengths correlating heights positively, the averages of all female measurements were higher than those of obese women. Obese women's measurements were much larger than all other measurements of obese women except in heights and some lengths.

To provide size charts for obese women, size intervals and size designations were incorporated in previous studies and market research. $3 \mathrm{~cm}$ pitch and $5 \mathrm{~cm}$ pitch in girth measurements were applied to make size charts and bust girth, and only $5 \mathrm{~cm}$ pitch in height was used in the study. Waist girth, hip girth, and height were introduced as control dimensions of size designations. To prove optimal size numbers, 3 observation rates were examined.

In the case of size chart for outerwear covering the upper body with $5 \mathrm{~cm}$ pitch, 95-95-155 (8.6\%) was the most frequently-observed size interval, and 9090-145 was the second highest interval.
In the case of size chart for outerwear covering the upper body with $3 \mathrm{~cm}$ pitch, the cell with the highest observation rate was $88-88-145$, and the percentage was $7.4 \%$. Next in order was 94-97-155, with 3.8\%.

According to the size charts with $5 \mathrm{~cm}$ pitch for outerwear covering the lower body, the highest observed cell was 85-95 (12.8\%). The second highest was 80$95(12.2 \%)$, and the third highest was $85-90(11.8 \%)$.

Based on the size chart with $3 \mathrm{~cm}$ pitch, the cell with the highest observation rate was $82-88(8.4 \%)$. Next in order was 82-97 (6.2\%) and 82-100 (4.6\%).

The most efficient size charts were provided by comparing the observed cells of over $1 \%, 2 \%$ and $3 \%$, from the aspects of coverage \& coverage efficiency. The highest coverage efficiency was confirmed by $5 \mathrm{~cm}$ size interval and observation rate of over $3 \%$ in both outerwear covering the upper body and lower body.

According to the comparison of coverage efficiency, a total of 10 size codes for outerwear covering the upper body were suggested. Bust girth, hip girth, and height were given as control dimensions, and 12 measurements were provided as referable dimensions.

Based on the comparison of coverage efficiency, 9 size codes were suggested for outwear covering the lower body. Waist girth and hip girth were introduced as control dimensions and 11 referable dimensions were also given.

KS Apparel Sizing System only provides fundamental size charts focusing on normal-sized women. It has to expand the size category from general to specific groups. In considering the adults' tendency toward obesity, reasonable and cost-efficient sizing system for the obese population is absolutely necessary. These size charts will be very helpful in manufacturing plus-sized garments in the fashion industry. In future studies, effectiveness of the study will be evaluated through wearing test of garments made by these size charts.

\section{References}

Ha, H. (2008). An analysis of the present condition of sizing system and fitting on formal pants of the on-line shopping mall for plus-sized women. Journal of the Korean 
Society of Costume, 58(1), 133-150.

Ha, H. (2009). An analysis of the fitting of plus-sized women's formal jackets in on-line shopping malls. The Research Journal of Costume Culture, 17(2), 203-215.

Hubert, H. B., Feinleip, M., MeNamara, P. M., \& Castelli, W. P. (1983). Obesity as an independent risk factor for cardiovascular disease: A 26-year follow-up of participants in the Framingham heart study. Circulation, 63, 968-977.

Jeon, J., \& Sung, S. (2004). Body fat correlation on phyiscal measurement of women in age group between $20 \mathrm{~S}$ and 30S. Journal of the Korean Society for Clothing Industry, 6(5), 641-647.

Kim, H. (2007a). A study on body shapes using 3D measurements and torso pattern of obese women. Doctoral dissertation, Sungshin Women's University, Seoul.

Kim, H. (2007b). Apparel sizing system for women with obese figure-Focusing on the formal dress for 20 s and 30s women-. Master's thesis, Hanyang University, Seoul.

Kim, J., \& Lee, J. (2008). A study on the somatotype of women in their twenties by degree of obesity and classification of silhouette. Korean Journal of Community Living Science, 19(3), 419-429.

Korean Agency for Technology \& Standards. (2004a). KS K 0055 sizing systems for elderly women's garments. Seoul: KSA.

Korean Agency for Technology \& Standards. (2004b). KS K 0051 sizing systems for female adult's garments. Seoul:
KSA.

Korean Agency for Technology \& Standards. (2004c). KS K 7003 garment construction and anthropometric surveys-body dimensions. Seoul: KSA.

Korean Agency for Technology \& Standards. (2004d). The $5^{\text {th }}$ Size Korea Survey final report. Seoul: Korean Agency for Technology \& Standards.

National Health Insurance Corporation. (2009). 2007 report of Korean health and nutrition survey. Seoul: NHIC.

Seok, H., \& Kim, I. (2003a). A study on the fit preference tendency for ready-to-wear by the age and obesity level of adult women. Journal of KHEA, 41(8), 63-77.

Seok, H., \& Kim, I. (2003b). A study on the fit preference tendency for ready-to-wear by the age and obesity level of adult women. Journal of KHEA, 41(9), 17-29.

Sung, M., \& Kim, H. (2001). Classification of the somatotype by obesity indexes and body cognition of female college students. Journal of the Korean Society for Clothing Industry, 3(3), 227-234.

Yi, K. (2009). A comparative study on establishing obesity judgment criteria for body characteristics analysis in Korean obese women. 2009 Fall KSCT proceedings, 250.

Yi, K., \& Kim, H. (2008). A suggestion of sizing system for developing taekwondo protectors. Journal of the Korean Society of Clothing and Textiles, 32(9), 1397-1406.

Yoon, J. (2007). Apparel sizing system for obese men in the 20 s-Focusing on casual wear-. Master's thesis, Hanyang University, Seoul.

\section{요 약}

본 연구는 20세 이상 비만여성을 위한 의류치수체계를 제안하기 위한 것으로, 2003 2004년 한국인 인 체치수조사사업의 원시자료 중 선행연구에 의해 의류학 분야에서 주로 사용되는 비만판정지수 및 비만판 정용 측정치 7개 항목(Rohrer지수, Vervaeck지수, BMI, 상대체중, 허리둘레, WHR, 젖가슴둘레) 중 5개 이 상의 비만판정도구에서 비만으로 판정된 499명을 대상으로 하였다. 연구의 결과는 다음과 같다. 치수체 계 제안에 앞서 2,212 명의 성인여성 전체의 인체치수와 499 명의 비만여성의 기초통계치수를 제시한 결과, 키 등의 높이항목 전체와 키와 상관관계가 높은 몇몇 길이항목을 제외한 대부분의 치수항목 즉, 둘레항 목, 두께 및 너비항목 등에서의 인체측정치가 크고, 비만판정지수도 높은 것으로 나타났다. 상의류의 치 수체계 제안을 위해 젖가슴둘레, 엉덩이둘레, 키가 기본신체부위로 선정되었으며, 하의류의 치수체계 제 안을 위해 허리둘레와 엉덩이둘레가 기본신체부위로 선정되었다. 치수체계 제안을 위한 교차분석표에서 젓가슴둘레, 허리둘레, 엉덩이둘레 등 둘레항목은 $3 \mathrm{~cm}$ 간격과 $5 \mathrm{~cm}$ 간격을 비교하여 제시하였으나 키는 $5 \mathrm{~cm}$ 간격으로만 한정하였다. 두 치수간격 중 보다 높은 커버율과 커버효율을 보이는 치수간격을 선택하 고, 사용자의 분포율도 전체 비만여성의 $1 \%$ 이상, $2 \%$ 이상 및 $3 \%$ 이상으로 나누어 각각의 분포율별로 커버율과 커버효율이 높은 치수구간을 선정하였다. 상의류와 하의류 치수체계 모두에서 $3 \%$ 이상의 사용 자 분포율을 보이는 $5 \mathrm{~cm}$ 치수간격이 가장 높은 커버효율을 나타냈으므로, 이 결과를 바탕으로 상의류를 위한 치수호칭으로 10 개, 하의류를 위한 치수호칭으로 9 개를 제안하였으며, 각 호칭별로 기본신체부위의 평균 인체측정치와 참고신체부위의 평균 인체측정치를 제공하여 호칭별 의류제품의 설계에 기초자료로 활용할 수 있도록 하였다. 